\title{
Sub-Picomolar Detection of SARS-CoV-2 RBD via Computationally-Optimized Peptide Beacons
}

\author{
Soumya P. Tripathy, 1,2,* Manvitha Ponnapati, 1,2,* \\ Joseph Jacobson, ${ }^{1,2}$ Pranam Chatterjee, ${ }^{1,2, \dagger}$ \\ ${ }^{1}$ Center for Bits and Atoms, ${ }^{2}$ Media Lab, \\ Massachusetts Institute of Technology \\ *These authors contributed equally. \\ †Corresponding author: pranam@mit.edu
}

The novel coronavirus SARS-CoV-2 continues to pose a significant global health threat. Along with vaccines and targeted therapeutics, there is a critical need for rapid diagnostic solutions. In this work, we employ deep learning-based protein design to engineer molecular beacons that function as conformational switches for high sensitivity detection of the SARS-CoV-2 spike protein receptor binding domain (S-RBD). The beacons contain two peptides, together forming a heterodimer, and a binding ligand between them to detect the presence of S-RBD. In the absence of S-RBD (OFF), the peptide beacons adopt a closed conformation that opens when bound to the S-RBD and produces a fluorescence signal (ON), utilizing a fluorophore-quencher pair at the two ends of the heterodimer stems. Two candidate beacons, C17LC21 and C21LC21, can detect the SRBD with limits of detection (LoD) in the sub-picomolar range. 


\section{${ }_{21}$ Introduction}

22 As numerous countries are experiencing additional waves of COVID-19, rapid, point23 of-care diagnostic tests enable triage of symptomatic individuals and control the out${ }_{24}$ breaks of the disease. The most widely employed diagnostic tests for SARS-CoV-2 are 25 reverse transcription-polymerase chain reaction (RT-PCR)-based methods (1), though

\section{We envision that these beacons can be easily integrated with on- chip optical sensors to construct a point-of-care diagnostic platform for SARS-CoV-2.}

other technologies based on CRISPR and loop-mediated amplification have been deployed as well (2-5). The best-in-class FDA authorized diagnostics, such as RT-PCR, have limits of detection (LoD) of $10^{2}-10^{3}$ RNA copies/ml, which is about 1-10 attomolar (aM) RNA in the test volume (6). RT-PCR tests, however, require laborious and expensive nucleic acid isolation, purification, and processing steps, which increases both the turnaround time of detection and the cost of testing $(6,7)$. Alternatively, there are FDA-authorized low-sensitivity, inexpensive, and rapid diagnostics. These tests, which often rely on antigen detection, have LoDs of $10^{5}-10^{7}$ RNA copies/ml, or around 1-100 femtomolar (fM) (8).

Recently, there has been significant effort to detect SARS-CoV-2 via fluorescence-based readouts to allow for specific signal amplification (9-12). Such methods largely rely on binding to SARS-CoV-2 RNA or DNA, which requires isolation of nucleic acids, as described above. In this study, we develop a molecular assay to detect the spike protein receptor binding domain $(\mathrm{S}-\mathrm{RBD})$ of SARS-CoV-2 using computationally-validated peptide beacons, which enable single-step detection of S-RBD presence through the production of a fluorescence signal. Our eventual goal is to integrate these optimized beacons 
42 within miniaturized total internal reflection fluorescence (TIRF) microscopes, which pro-

43 vide exquisite sensitivity by exciting fluorophores present within nanometer proximity of

44 the device surface (13), producing high signal-to-background ratios and enabling rapid

45 and ultra-sensitive detection of SARS-CoV-2.

\section{${ }_{46}$ Results}

\section{${ }_{47}$ Engineering of Peptide Beacon Architecture}

48 Our molecular beacon design includes two heterodimer-forming peptides, a binding ligand

49 to the S-RBD, as well as a fluorophore-quencher pair at the terminal ends of the beacon

50 (Figure 1A). This fluorophore-quencher pair induces fluorescence quenching through the 51 mechanism of Förster resonance energy transfer (FRET), where the efficiency of energy 52 transfer between the fluorophore and quencher is proportional to their spatial distance.

53 Hence, a small change in spatial distance between the two beacon arms can drastically 54 change the FRET efficiency, thus affecting the fluorescent quantum yield of the fluo55 rophore. Here, we utilized a commonly-used fluorophore-quencher pair: fluorescein isoth${ }_{56}$ iocyanate (FITC) and [4-(N,N-dimethylamino)phenylazo] benzoyl (DABCYL), respec57 tively. Upon binding to the target protein, the fluorophore and quencher are separated 58 enough to observe an increase in fluorescence signal proportional to the amount of S-RBD 59 present (Figure 1B).

\section{${ }_{60}$ Computational Selection of Peptide Beacon Candidates}

${ }_{61}$ We utilized the coiled-coil peptide beacons designed by Mueller, et al. (14). Mueller, 62 et al., employed the parallel heterodimer reported by Thomas, et al., to design acidic ${ }_{63} 21$ mer peptide portions of a beacon detecting CREB binding protein (15). The reported 64 peptide beacon designs consisted of the 21 mer conjugated with a binding ligand and one 
65 of the three basic peptides: C13, C17, C21 (14). We designed our peptide beacons by

66 replacing the binding portion of the sequences designed by Grossman with our previously

67 engineered 23mer peptide that can bind to S-RBD and induce its degradation via the 68 ubiquitin-proteasomal pathway (16).

69

70 To computationally verify that insertion of our $23 \mathrm{mer}$ peptide confers S-RBD bind71 ing capability to our peptide beacon sequences, we folded and docked the three de72 signs (C13LC21, C17LC21, C21L21) using trRosetta, Rosetta, and HDOCK (Figure 73 2A) (17-19). Our results show that in the absence of S-RBD, all three peptides show 74 terminal ends of the beacons, representing the fluorophore and the quencher, in close 75 proximity to each other $(\mathrm{OFF})$ in at least one of the top predicted models from tr76 Rosetta (17) or Rosetta Abinitio (18) (Figure 2B-2C). Alternatively, when the peptide 77 beacon sequences were docked against S-RBD utilizing HDOCK (19), we observed the 78 terminal ends to be spatially distanced from each other, indicating a possible ON state 79 (Figure 2D). These results motivated us to test these three peptide beacon designs exper80 imentally.

\section{Validation of S-RBD Binding in Human Cells}

82 To rapidly validate the binding capability of our peptide beacon designs, we adapted 83 our previously-described degradation assay in human cells, by fusing our peptide beacon 84 candidates to the CHIP $\triangle \mathrm{TPR}$ E3 ubiquitin ligase, which can tag target proteins for degra85 dation via the ubiquitin-proteasomal pathway in human cells. (16). After co-transfection 86 with a plasmid expressing S-RBD C-terminally fused to superfolder GFP (sfGFP), we can 87 measure binding affinity to S-RBD as relative to sfGFP degradation. We tested various 88 peptide beacon combinations, employing our validated mutant S-RBD-binding peptide 
derived from ACE2, A2N (16), as well as a positive nanobody control, which has shown high affinity to S-RBD (20). As expected, these moieties alone demonstrate robust degradation capabilities, while the arms-only negative controls (C13, C17, C21, and C21*) show negligible degradation, hence no binding to the S-RBD. Of the complete peptide beacon constructs, the $\mathrm{C} 17-\mathrm{A} 2 \mathrm{~N}-\mathrm{C} 21^{*}(\mathrm{C} 17 \mathrm{LC} 21)$ beacon induces the greatest level of degradation of S-RBD-sfGFP, followed by C21LC21 and C13LC21, the latter of which exhibited minimal degradation capabilities (Figure 3A).

\section{In vitro Detection of S-RBD}

After ascertaining the binding capability of our candidates in human cells, we characterized the response of the peptide beacons in vitro in the presence of S-RBD. As a negative control, we also measured the cross reactivity of our peptide beacons towards Hemagglutinin protein of Influenza A H3N2 (HA) (21). We first purified peptide beacons (CxxLC21) from the reaction mixture of $\mathrm{CxxL}+\mathrm{C} 21$, using high performance liquid chromatography (HPLC) and confirmed the presence of CxxLC21 in the collected fraction from HPLC through MALDI-TOF mass spectrometry (Supplementary Figure 1). We measured the fluorescent signals from the peptide beacons following a 10 minute exposure to different concentrations of S-RBD and HA in 1X PBS and pH 7.4 (Figure 3B-D). Of the three peptide beacons, C17LC21 showed highest sensitivity towards S-RBD with a LoD of nearly $20 \mathrm{fM}\left(K_{d}=1.615 \times 10^{-12}\right)$, followed by C21LC21 having an LoD of 400 fM $\left(K_{d}=6.766 \times 10^{-13}\right)$. However, C13LC21 showed negligible response towards both S-RBD and HA. In conclusion, C17LC21 and C21LC21 are able to detect the presence of S-RBD with sub-picomolar sensitivity and low cross-reactivity, thus motivating their application for rapid detection of SARS-CoV-2. 


\section{${ }_{112}$ Discussion}

121

In this study, using existing deep learning tools for protein structure prediction and energy-based modeling suites, we designed and tested a set of molecular beacons that can potently bind to the S-RBD and release a fluorescence signal via FRET, enabling sub-picomolar detection levels. Integration of these peptide beacons within optical sensors, such as miniature TIRF microscopes, may reduce the LoD to sub-femtomolar level, thus yielding a rapid, point-of-care diagnostic platform for SARS-CoV-2. Such a diagnostic would be faster than existing RT-PCR assays and more sensitive than antigen-based rapid testing.

Our pipeline also showcases a use case for current deep learning tools for protein structure prediction in a protein design pipeline. Using this approach, molecular beacons can be designed to detect protein targets within other viral species. In addition, by using existing protein structure prediction tools to get rapid insights into the structure of the protein, it may be possible to design an entire peptide beacon sequence from scratch even in the absence of a known binding partner. By fixing the heterodimer motif, binding loops of molecular beacons can be filled in via protein hallucination, using tools like trRosetta (22). Thus, by employing a hybrid approach of state-of-the-art protein modeling tools and robust experimental validation, our molecular beacon design pipeline serves as a powerful platform to fight COVID-19 and future emergent viral threats. 
132

133

134

135

136

137

138

139

140

141

142

143

144

145

146

147

148

149

\section{Materials and Methods}

\section{In silico Selection of Candidate Peptide Beacons}

The three peptide beacon sequences were folded in the absence of S-RBD using trRosetta. trRosetta is a deep learning tool to predict structures from sequence information (17).

The three peptide beacon sequences were also folded using Rosetta Abinitio folding. Abinitio folding solves the protein structure from sequence through physics-based constraints rather than relying on previously solved structures like trRosetta (18).

The three peptide beacon sequences were docked against the S-RBD using HDOCK. HDOCK is a protein-protein docking platform that combines ab-initio docking and templatebased modeling (19). The top 100 predictions from HDOCK were analyzed to visualize the structure of peptide beacon sequences in the presence of S-RBD.

\section{Generation of Plasmids}

pcDNA3-SARS-CoV-2-S-RBD-sfGFP (Addgene \#141184) and pcDNA3-R4-uAb (Addgene \#101800) were obtained as gifts from Erick Procko and Matthew DeLisa, respectively. Peptide beacon sequences were ordered as gBlocks (IDT), and were amplified with overhangs for Gibson Assembly-mediated insertion into linearized pcDNA3-R4-uAb digested with HindIII and EcoRI. Assembled constructs were transformed into $50 \mu \mathrm{L}$ NEB Turbo Competent E. coli cells, and plated onto LB agar supplemented with the appropriate antibiotic for subsequent sequence verification of colonies and plasmid purification.

\section{Cell Culture}

HEK293T cells were maintained in Dulbecco's Modified Eagle's Medium (DMEM) supplemented with 100 units/mL penicillin, $100 \mathrm{mg} / \mathrm{mL}$ streptomycin, and $10 \%$ fetal bovine 
serum (FBS). RBD-sfGFP (250 ng) and peptide-E3 ligase fusion (250 ng) plasmids were transfected into cells $\left(2 \times 10^{5} /\right.$ well in a 24-well plate) with Lipofectamine 3000 (Invitrogen) in Opti-MEM (Gibco). After 5 days post-transfection, cells were harvested and analyzed on a BD FACSCelesta flow cytometer (BD Biosciences) for GFP fluorescence (488-nm laser excitation, 530/30 filter for detection). Cells expressing GFP were gated, and percent GFP + depletion to the RBD-sfGFP only control were calculated. All samples were performed in independent transfection duplicates $(n=2)$, and percentage depletion values were averaged. Standard deviation was used to calculate error bars.

\section{Peptide Synthesis and Purification}

CxxL (25 $\mu \mathrm{M}, 1 \mathrm{X}$ PBS, pH 7.4) was added to C21 (25 $\mu \mathrm{M}, 1 \mathrm{X}$ PBS, pH 7.4) and incubated at room temperature for 2 hours for the conjugation reaction between the thiol group of Cysteine at N-terminus of $\mathrm{CxxL}$ and the maleimide group at the N-terminus of $\mathrm{C} 21$ (Figure 1C). CxxL+C21 is used to represent the reaction mixture obtained after 2 hours of reaction between $\mathrm{CxxL}$ and $\mathrm{C} 21$ in $1 \mathrm{X}$ PBS, pH 7.4 at room temperature. MALDI-TOF mass spectroscopy of $\mathrm{CxxL}+\mathrm{C} 21$ confirms the presence of $\mathrm{CxxLC} 21$ in $\mathrm{CxxL}+\mathrm{C} 21$ along with CxxL and C21 (Supplementary Figure 1A-1C). The reaction mixture of CxxL and C21 contains the mixture of individual CxxL, C21, and CxxLC21 after 2 hours of reaction in 1X PBS, pH 7.4 at room temperature. Consequently, CxxLC21 from CxxL+C21 was purified using high performance liquid chromatography (HPLC) (Agilent 1100) using a C14 H31 column. HPLC chromatogram of CxxL+C21 contains peaks at distinct retention time than that of CxxL and C21 in HPLC chromatogram (Supplementary Figure 1D-1F), which are considered as the fraction containing CxxLC21. The fraction was collected and MALDI-TOF mass spectroscopy was performed (Microflex LRF, Bruker), confirming the presence of CxxLC21 at higher concentration than that of CxxL and C21 (Supplementary 
Figure 1G-1I). The collected fraction was freeze-dried and used for detection of S-RBD and HA.

\section{In vitro Detection of S-RBD}

S-RBD (Abcam ab273065) and Influenza H3N2 hemaglutinin (HA) (MyBioSource MBS5308351) were titrated against purified CxxLC21. S-RBD and HA proteins were serially diluted in 1X PBS, pH 7.4 from nanomolar (nM) to attomolar (aM) concentration. $2 \mathrm{nM}$ of CxxLC21 in 1X PBS, pH 7.4 ( $\mathrm{n}=3$ or 5) was exposed to different concentrations of the target protein (S-RBD or HA) and incubated for 10 minutes at room temperature. Fluorescence intensity was subsequently measured using a Tecan Spark well plate reader at excitation and emission wavelength of $470 \mathrm{~nm}$ and $525 \mathrm{~nm}$, respectively. The fluorescence data was fitted to one site total binding with saturation curve in the Prism software using the following equation:

$$
Y=\frac{\operatorname{Bmax} * X}{K_{d}+X}+N S * X+\text { Background }
$$

where $Y$ is the fraction of peptide beacon bound to target, Bmax is the maximum binding in units of $Y, X$ is the concentration of target, $K_{d}$ is the equilibrium dissociation constant in units of $X$, and $N S$ is the slope of the non-linear regression. The fraction of peptide beacon bound to target $(Y)$ is calculated by normalizing fluorescent intensities obtained at different $(X)$ by considering highest fluorescence intensity obtained at maximum SRBD concentration as hundred percent. The limit of detection (LoD) is estimated from the equation given below.

$$
L o D=Y_{\text {Blank }}+3 * S D_{\text {Blank }}
$$




\section{Statistics and Reproducibility}

All samples were performed in independent duplicates $(n=2)$, triplicates $(n=3)$, or quintuplicates $(\mathrm{n}=5)$, as indicated. Standard deviation was used to calculate error bars. Statistical analyses was performed using the two-tailed Student's t test, using the GraphPad software package.

\section{Acknowledgments}

We thank Dr. Neil Gershenfeld, Dr. Shuguang Zhang and Dr. Edward S. Boyden for shared lab equipment, and Teodora Stan for techical assistance. This work was supported by the consortia of sponsors of the MIT Media Lab and the MIT Center for Bits and Atoms, and by Jeremy and Joyce Wertheimer.

\section{Competing Interests}

P.C., M.P., S.P.T and J.M.J. are listed as inventors for U.S. Provisional Patent Application 63/182,537 entitled "Peptide Based Probes For the Detection of SARS-CoV-2."

\section{Author Contributions}

S.P.T. conducted peptide beacon purification, synthesis, and in vitro characterization. M.P. conducted computational design and docking protocols, and performed experiments. P.C. built constructs, performed degradation assays, and conducted data analysis. P.C., S.P.T, and M.P. wrote the manuscript. P.C. and J.M.J supervised the project.

\section{Data and Code Availability Statement}

All data needed to evaluate the conclusions in the paper are present in the paper. All „source computational and experimental data files can be accessed at: https://tinyurl. com/covidpepbeacons. 


\section{${ }_{220}$ References}

1. Wang, W. et al. Detection of SARS-CoV-2 in different types of clinical specimens. JAMA (2020).

2. Patchsung, M. et al. Clinical validation of a cas13-based assay for the detection of SARS-CoV-2 RNA. Nat Biomed Eng 4, 1140-1149 (2020).

3. Guo, L. et al. SARS-CoV-2 detection with CRISPR diagnostics. Cell Discov 6 (2020).

4. Broughton, J. P. et al. CRISPR-cas12-based detection of SARS-CoV-2. Nat Biotechnol 38, 870-874 (2020).

5. Mautner, L. et al. Rapid point-of-care detection of SARS-CoV-2 using reverse transcription loop-mediated isothermal amplification (RT-LAMP). Virology Journal 17 (2020).

6. Kevadiya, B. D. et al. Diagnostics for SARS-CoV-2 infections. Nature Materials 20, 593-605 (2021).

7. Bustin, S. A. \& Nolan, T. RT-qPCR testing of SARS-CoV-2: A primer. International Journal of Molecular Sciences 21, 3004 (2020).

8. Corman, V. M. et al. Comparison of seven commercial SARS-CoV-2 rapid point-ofcare antigen tests: a single-centre laboratory evaluation study. The Lancet Microbe (2021).

9. Bokelmann, L. et al. Point-of-care bulk testing for SARS-CoV-2 by combining hybridization capture with improved colorimetric LAMP. Nature Communications 12 (2021). 
10. Xun, G., Lane, S. T., Petrov, V. A., Pepa, B. E. \& Zhao, H. A rapid, accurate, scalable, and portable testing system for COVID-19 diagnosis. Nature Communications $12(2021)$.

11. Ding, X. et al. Ultrasensitive and visual detection of SARS-CoV-2 using all-in-one dual CRISPR-cas12a assay. Nature Communications 11 (2020).

12. Kellner, M. J., Koob, J. G., Gootenberg, J. S., Abudayyeh, O. O. \& Zhang, F. SHERLOCK: nucleic acid detection with CRISPR nucleases. Nature Protocols 14, 2986-3012 (2019).

13. Wazawa, T. \& Ueda, M. Total internal reflection fluorescence microscopy in single molecule nanobioscience. In Microscopy Techniques, 77-106 (Springer Berlin Heidelberg, 2005). URL https://doi.org/10.1007/b102211.

14. Mueller, C. \& Grossmann, T. N. Coiled-coil peptide beacon: A tunable conformational switch for protein detection. Angewandte Chemie International Edition 57, 17079-17083 (2018).

15. Thomas, F., Boyle, A. L., Burton, A. J. \& Woolfson, D. N. A set ofde NovoDesigned parallel heterodimeric coiled coils with quantified dissociation constants in the micromolar to sub-nanomolar regime. Journal of the American Chemical Society 135, 5161-5166 (2013).

16. Chatterjee, P. et al. Targeted intracellular degradation of SARS-CoV-2 via computationally optimized peptide fusions. Communications Biology 3 (2020).

17. Yang, J. et al. Improved protein structure prediction using predicted interresidue orientations. Proceedings of the National Academy of Sciences 117, 1496-1503 (2020). 
18. Bonneau, R. et al. Rosetta in CASP4: Progress in ab initio protein structure prediction. Proteins: Structure, Function, and Genetics 45, 119-126 (2001).

19. Yan, Y., Tao, H., He, J. \& Huang, S.-Y. The HDOCK server for integrated protein-protein docking. Nature Protocols 15, 1829-1852 (2020).

20. Xiang, Y. et al. Versatile and multivalent nanobodies efficiently neutralize SARSCoV-2. Science eabe4747 (2020).

21. Russell, C. J., Hu, M. \& Okda, F. A. Influenza hemagglutinin protein stability, activation, and pandemic risk. Trends in Microbiology 26, 841-853 (2018).

22. Anishchenko, I., Chidyausiku, T. M., Ovchinnikov, S., Pellock, S. J. \& Baker, D. De novo protein design by deep network hallucination (2020). 


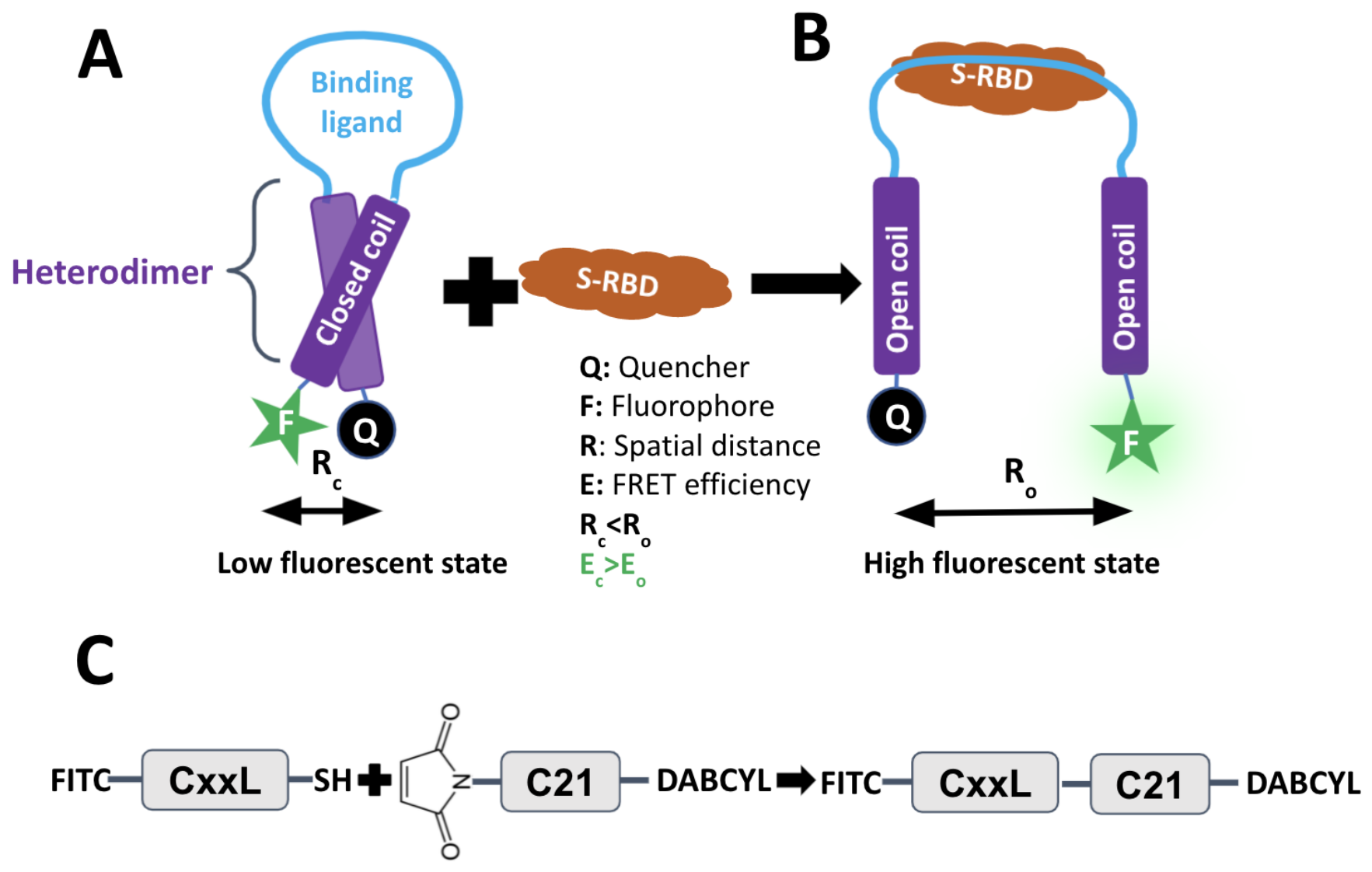

Figure 1: Engineering of peptide beacon architecture. A) Low-fluorescent state is the closed heterodimer state of the peptide beacon in the absence of S-RBD. B) High-fluorescent state is the opencoil state after binding of S-RBD with the loop of the peptide beacon. C) CxxL (25 uM, 1X PBS, pH 7.4) was added to C21 (25 uM, 1X PBS, pH 7.4) and incubated at room temperature for 2 hours for the conjugation reaction between the thiol groups of cysteine at the N-terminus of CxxL and the maleimide on the N-terminus of $\mathrm{C} 21$. 


\section{A}

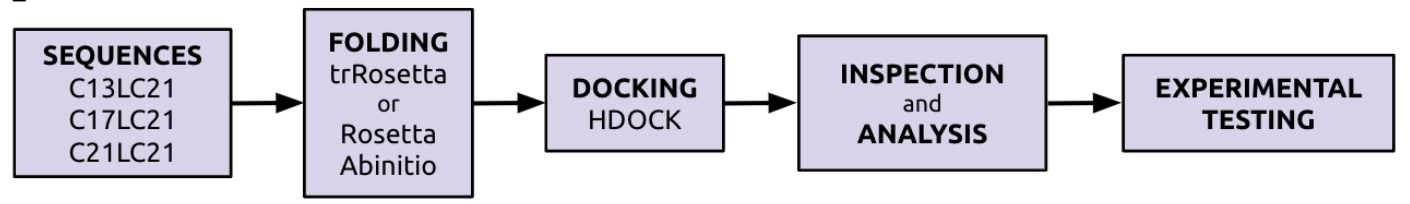

B

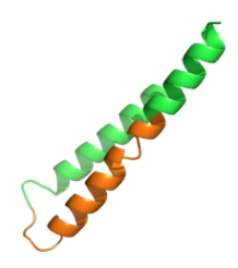

C13LC21

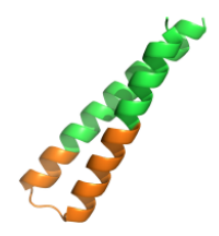

C13LC21

D

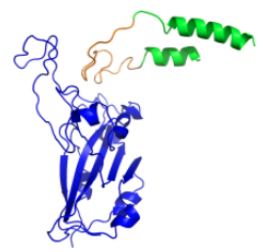

C13LC21

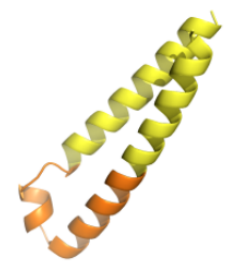

C17LC21

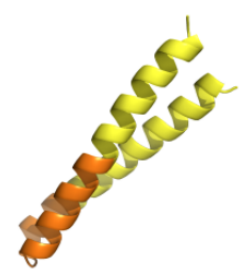

C17LC21

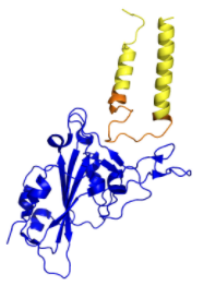

C17LC21

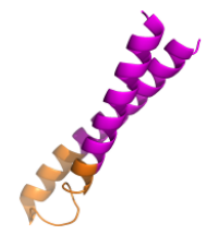

C21LC21

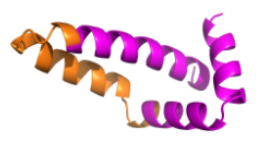

C21LC21

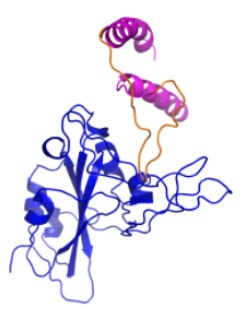

C21LC21

Figure 2: In silico verification of peptide beacon architecture. A) Validation pipeline. Sequences were folded ab initio via trRosetta or Rosetta Abinitio. The top structures were docked to S-RBD via HDOCK. Docked structures were analyzed and candidate beacons were chosen. Highest scoring B) trRosetta structures C) Rosetta Abinitio structures and D) Docked HDOCK structures of the three candidate beacons. The conserved binding peptide moiety is highlighted in orange. S-RBD (derived from PDB 6MOJ) is indicated in blue. 
A

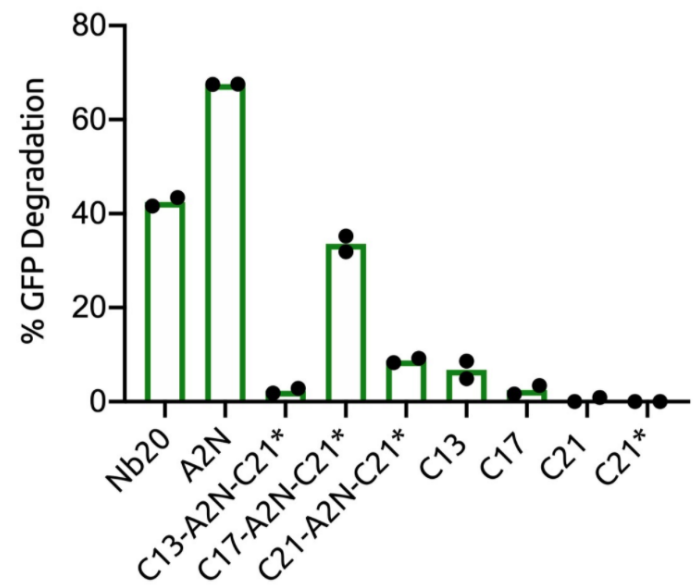

\section{C17LC21}

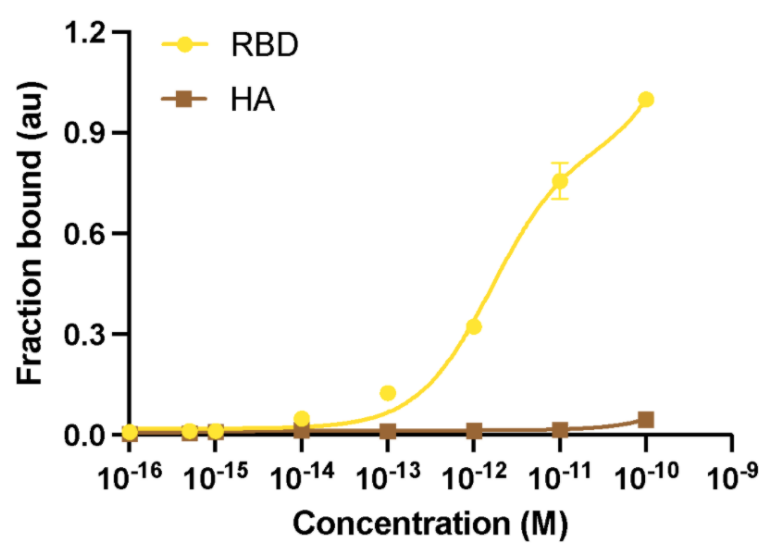

B

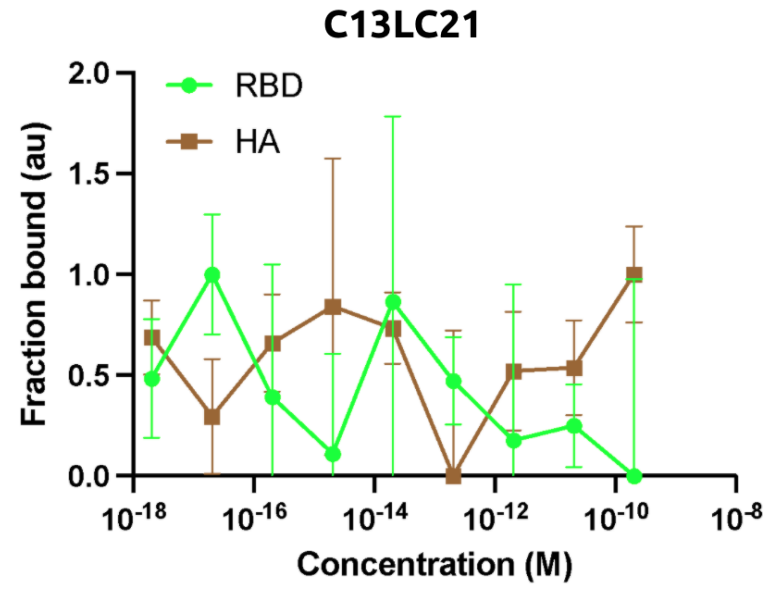

D

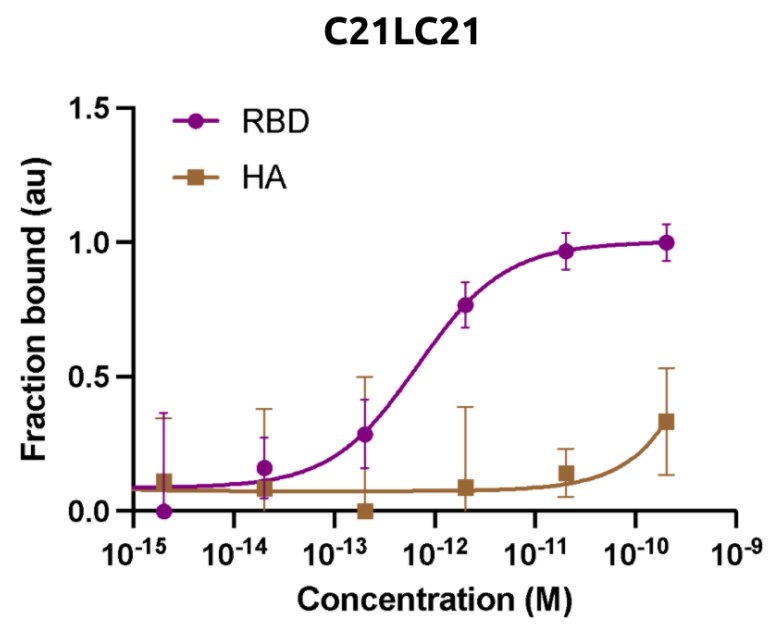

Figure 3: Experimental characterization of peptide beacons. A) Analysis of S-RBD-sfGFP degradation by flow cytometry. All samples were performed in independent transfection duplicates $(n=2)$ and gated on GFP + fluorescence. Mean percentage of GFP + cell depletion was calculated in comparison to the S-RBD-sfGFP only control. C13LC21 is referred to as C13-A2N-C21*, C17LC21 is referred to as $\mathrm{C} 17-\mathrm{A} 2 \mathrm{~N}-\mathrm{C} 21^{*}$, and $\mathrm{C} 21 \mathrm{LC} 21$ is referred to as $\mathrm{C} 21-\mathrm{A} 2 \mathrm{~N}-\mathrm{C} 21^{*}$. Titration of the target recombinant S-RBD and Influenza H3N2 (HA) with $2 \mathrm{nM}$ of HPLC-purified B) C13LC21 (n=3), C) C17LC21 (n=5), D) $\mathrm{C} 21 \mathrm{LC} 21(\mathrm{n}=3)$ in $1 \mathrm{X}$ PBS, $\mathrm{pH}$ 7.4. 

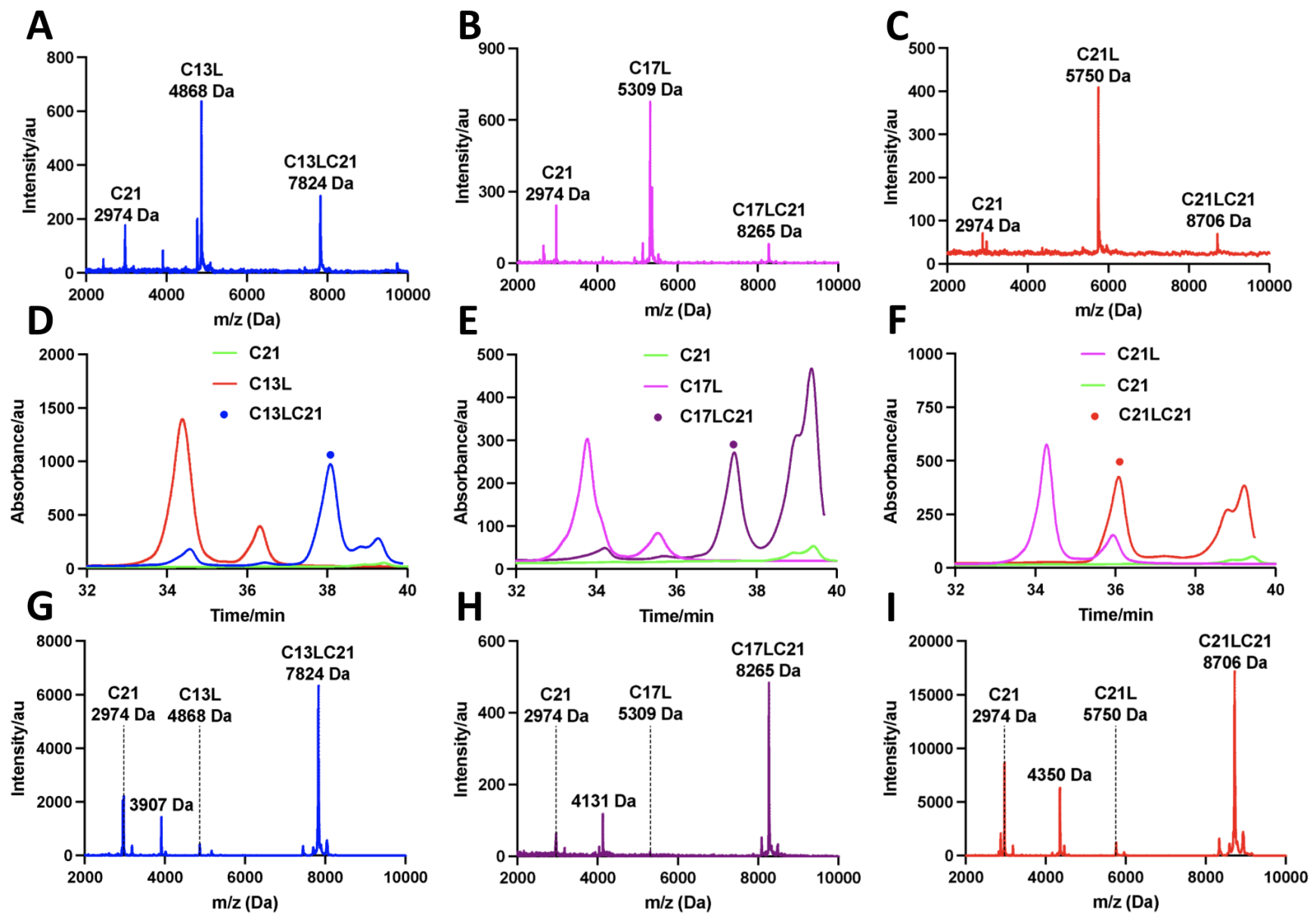

Figure S1: Purification and synthesis of peptide beacons. A-C) MALDI-TOF mass spectrum of CxxL+C21 shows peaks corresponding to C21, CxxL, and CxxLC21. D-F) HPLC chromatograms of CxxLC21, plotted with HPLC chromatograms of CxxL and C21. The chromatogram of C13L+C21, $\mathrm{C} 17 \mathrm{~L}+\mathrm{C} 21$, and $\mathrm{C} 21 \mathrm{~L}+\mathrm{C} 21$ shows the appearance of new peaks having retention times of $38 \mathrm{~min}, 37 \mathrm{~min}$, and 36 min, respectively (new peaks marked in colored dot in D-E). Fraction of material corresponding to new peaks was collected during HPLC. G-I) MALDI-TOF mass spectrum of collected fraction from HPLC of CxxL+C21 shows dominant peak corresponding to CxxLC21 and negligible peaks corresponding to $\mathrm{CxxL}$ and $\mathrm{C} 21$. CxxL+C21 represents the reaction mixture of CxxL and $\mathrm{C} 21$ after 2 hours of reaction at room temperature. CxxLC21 represents the peptide beacons. 